\title{
Expert consensus on acute exacerbation of chronic obstructive pulmonary disease in the People's Republic of China
}

This article was published in the following Dove Press journal:

International Journal of COPD

25 April 2014

Number of times this article has been viewed

\author{
Bai-qiang $\mathrm{Cai}^{\prime}$ \\ Shao-xi $\mathrm{Cai}^{2}$ \\ Rong-chang Chen ${ }^{3}$ \\ Li-ying Cui ${ }^{4}$ \\ Yu-lin Feng ${ }^{5}$ \\ Yu-tong $\mathrm{Gu}^{6}$ \\ Shao-guang Huang ${ }^{7}$ \\ Rong-yu Liu ${ }^{8}$ \\ Guang-nan Liu ${ }^{9}$ \\ Huan-zhong Shi ${ }^{10}$ \\ Yi Shi" \\ Yuan-lin Song ${ }^{6}$ \\ Tie-ying Sun ${ }^{12}$ \\ Chang-zheng Wang ${ }^{13}$ \\ ling-lan Wang \\ Fu-qiang Wen \\ Wei Xiao ${ }^{14}$ \\ Yong-jian $\mathrm{Xu}^{15}$ \\ Xi-xin Yan $^{16}$ \\ Wan-zhen $\mathrm{Yao}^{17}$ \\ Qin $Y u^{18}$ \\ Jing Zhang ${ }^{6}$ \\ Jin-ping Zheng ${ }^{3}$ \\ Jie Liu ${ }^{6}$ \\ Chun-xue Bai ${ }^{6}$
}

'Department of Pulmonary Medicine, Peking Union Medical College Hospital, Chinese Academy of Medical Sciences and Peking Union Medical College, Beijing, ${ }^{2}$ Southern Medical University South Hospital, Guangzhou, ${ }^{3}$ The First Affiliated Hospital of Guangzhou Medical College and Guangzhou Institute of Respiratory Diseases, Guangzhou, ${ }^{4}$ Affiliate Hospital of Inner Mongolia Medical University, Huhehaote, ${ }^{5}$ Huaxi Hospital of Sichuan

University, Chendu, ${ }^{6}$ Zhongshan Hospital, Shanghai Medical College, Fudan University and Shanghai Respiratory

Research Institute, Shanghai, ${ }^{2}$ Ruijing Hospital of Shanghai Jiaotong University, Shanghai, ${ }^{8}$ The First Affiliated University of Anhui Medical University, Hefei, ${ }^{9}$ The First Affiliated University of Guangxi Medical University, Nanning, ${ }^{10}$ Beijing Chao-Yang Hospital, Capital Medical University, Beijing, "General Hospital of Nanjing Military Region, Nanjing, 'BBeijing Hospital of the Ministry of Health, Beijing, ${ }^{13}$ Xinqiao Hospital, Third Military Medical University, Chongqing, ${ }^{14} \mathrm{Q}$ Qilu Hospital of Shandong University, Jinan, ${ }^{15}$ Tongji Hospital, Tongji Medical College, Huazhong University of Science and Technology, Wuhan, HeThe Second Affiliated Hospital of Hebei Medical
${ }^{2}$ The University and Hebei Research Institute of Respiratory Medicine, Shijiazhuang, ${ }^{17}$ The Third Affiliated Hospital of Beijing University, Beijing, ${ }^{18}$ The First Affiliated University of Lanzhou University, Lanzhou, People's Republic of Chin

Correspondence: Chun-xue Bai 180 Fenglin Road, Department of Pulmonary Medicine, Zhongshan

Hospital, Shanghai, 200032,

People's Republic of China

Email bai.chunxue@zs-hospital.sh.cn
Abstract: Chronic obstructive pulmonary disease (COPD) is a common disease that severely threatens human health. Acute exacerbation of COPD (AECOPD) is a major cause of disease progression and death, and causes huge medical expenditures. This consensus statement represents a description of clinical features of AECOPD in the People's Republic of China and a set of recommendations. It is intended to provide clinical guidelines for community physicians, pulmonologists and other health care providers for the prevention, diagnosis, and treatment of AECOPD.

Keywords: COPD, AECOPD, recommendations, guidelines

\section{Introduction}

Chronic obstructive pulmonary disease (COPD) is a common disease that severely threatens human health. Social and economic burden of COPD will rise to fifth, and COPD will become the third leading cause of death worldwide by $2020 .{ }^{1}$ A survey which included seven regions and 20,245 adults indicated that the prevalence of COPD in those over 40 years in the People's Republic of China was as high as $8.2 \% .^{2}$ On average, each COPD patient suffers from 0.5 to 3.5 episodes of acute exacerbations per year, and acute exacerbation of COPD (AECOPD) is a major cause of death and causes huge medical expenditures. In the United States, the inhospital mortality of AECOPD is about $4.3 \%$, and cost 9,545 USD per patient for hospitalization in $2006 .^{3}$ According to an epidemiological study, the cost of AECOPD for an inpatient in the People's Republic of China has been 11,598 RMB a year. ${ }^{4}$ Especially for patients whose hospital stay ends in death, their expenses, which are related to the high cost of life-support, increase remarkably. ${ }^{5}$ The prevention, early detection, and treatment of AECOPD is clinically significant because of its increasing social burden and negative impact on quality of life, pulmonary function, and disease progression.

\section{An overview of AECOPD}

AECOPD is an acute event characterized by a worsening of the patient's respiratory symptoms (typically dyspnea, cough, increased sputum volume, and/or sputum purulence) that is beyond normal day-to-day variations and leads to a change in medication. ${ }^{1}$ AECOPD is a clinical diagnosis of exclusion, made where no alternative specific cause (such as pneumonia, congestive heart failure, pneumothorax, pleural effusion, pulmonary embolism [PE], and arrhythmia) for deterioration has been identified by clinical examination and/or corroborative testing. The worsening of respiratory 
symptoms may or may not warrant a change in underlying therapy, and the symptoms will typically resolve over a period of days to weeks. ${ }^{1,6,7}$

The most common causes of AECOPD appear to be viral infections of the upper respiratory tract and bacterial infections of the trachea and bronchi. The bacterial burden increases during exacerbations, and new bacterial strains lead to specific immune responses and inflammation of neutrophils associated with AECOPD. Bacterial and viral infections and air pollution can induce acute exacerbations. Pulmonary infection or colonization of bacteria and viruses are always accompanied with exacerbations of airway inflammation. However, the causes of about one-third of AECOPD cases cannot be identified. The goals of treatment for AECOPD are to minimize the impact of the current exacerbation and prevent the development of subsequent exacerbations. ${ }^{1}$

Short-acting bronchodilators with or without short-acting anticholinergics are usually preferred bronchodilators for treatment of AECOPD. Systemic corticosteroids and antibiotics can shorten recovery time, improve lung function (forced expiratory volume in 1 second $\left[\mathrm{FEV}_{1}\right]$ ), alleviate hypoxemia and reduce the risk of early relapse and treatment failure, and reduce the length of hospital stay. Antiviral treatment is no longer recommended in AECOPD.

AECOPD can be prevented. Approaches that can reduce exacerbations and hospitalizations include but are not limited to the following: smoking cessation, influenza and pneumococcal vaccines, long-acting bronchodilators with or without inhaled corticosteroids, and phosphodiesterase-4 inhibitors. ${ }^{1,7}$

\section{Etiology of AECOPD}

The most common cause of AECOPD is respiratory infection. Viral or bacterial respiratory infection is identified in $78 \%$ of AECOPD patients. Other triggers for AECOPD include smoking, air pollution, inhaled allergens, surgery, sedative drugs, pneumothorax, pleural effusion, congestive heart failure, arrhythmia, and PE. To conclude, AECOPD can be precipitated by multiple factors; for example, viral infection and air pollution can aggravate airway inflammation and then secondary bacterial infection occurs. ${ }^{6,7}$

\section{AECOPD and viral infections}

It has been shown that AECOPD can be induced by viral upper respiratory tract infections, which occur in almost 50\% of patients with AECOPD. Rhinovirus, respiratory syncytial virus, and influenza viruses are common causes. Up to $64 \%$ of exacerbations are associated with a common cold, which is usually caused by rhinovirus occurring before exacerbation onset. Research shows that rhinovirus infection leads to significant proliferation of existing bacteria in the airways and increases bacterial load. It is suggested that rhinovirus infection can change flora in COPD patients' airways and is involved in secondary bacterial infection. ${ }^{8}$ Respiratory syncytial virus is another common agent, while AECOPD caused by influenza viruses is relatively infrequent. An increased prevalence of respiratory viruses in low temperature winter months accounts for the high incidence of AECOPD in winter each year. The symptoms of AECOPD caused by viral upper respiratory tract infections are more severe than those induced by bacteria. Also, the duration is much longer, and possibilities of relapse increase. Both neutrophils and eosinophils increase in sputum after viral infection. About $25 \%$ of patients suffer from mixed infection with viruses and bacteria which can lead to a critical condition and an increased length of hospital stay. ${ }^{9-11}$

\section{AECOPD and bacterial infections}

Bacteria are isolated from sputum using standard culture techniques in $40 \%-60 \%$ of exacerbations. The three most common species isolated in COPD exacerbations are Haemophilus influenzae, Moraxella catarrhalis, and Streptococcus pneumoniae, followed by other infectious agents including Pseudomonas aeruginosa, Gram-negative Enterobacteriacea, Staphylococcus aureus, and H. parainfluenzae. Recently, a multicenter study in the People's Republic of China demonstrated that the sputum culture results from 331 of 884 AECOPD patients were positive (37.4\%). ${ }^{12}$ Among them, $78.8 \%$ were Gram-negative bacteria in which Klebsiella pneumonia and $P$. aeruginosa were most commonly seen, and the next was $H$. influenzae; $15.5 \%$ were Gram-positive cocci including $S$. pneumoniae and Staphylococcus aureus. ${ }^{12}$ Bronchoscopic studies have shown that $25 \%$ of patients have bacteria colonization in their lower airways in stable COPD, while up to $50 \%$ of patients during exacerbations have bacteria in their lower airways. Smoking is an independent risk factor of bacteria colonization in lower airways, which can be implied by high levels of neutrophils, interleukin-8, and tumor necrosis factor alpha in bronchoalveolar lavage fluid or sputum of patients in a stable phase. These markers, representing airway inflammation, increase in AECOPD and decline when antibiotics are used. Antibiotics have a significant effect on exacerbations caused by infection, especially for Anthonisen I exacerbations with severe airflow limitation and resolution of symptoms (associated with increased sputum volume, sputum purulence, and severe dyspnea). ${ }^{11,12}$ 


\section{AECOPD and atypical pathogens}

Atypical pathogen is another factor that cannot be ignored. Chlamydia pneumoniae is currently considered an important trigger for exacerbations. Research has shown that 3\%-5\% of AECOPD is caused by C. pneumoniae. In one such study of AECOPD patients, $60.9 \%$ had an acute infection of C. pneumoniae, which was markedly higher than the control group (15.9\%), and for COPD patients in stable phase, the infection rate was $22.9 \% .^{11}$

\section{AECOPD and environmental factors}

Noninfectious factors such as smoking, air pollution, and inhaled allergens can also induce airway inflammation. These agents can cause mucosa edema, smooth muscle spasm, and increased secretions, which facilitate the growth of colonized bacteria. Epidemic data have shown that air pollutants, especially the concentration of particles with diameter of 2.5-10 $\mu \mathrm{m}$ (particle material [PM] 10, PM 2.5), are associated with the onset of AECOPD, and environment temperature drop can also induce AECOPD. In addition, there are causes of AECOPD yet to be identified. ${ }^{13}$

\section{Assessment of AECOPD Clinical manifestations}

Exacerbation of dyspnea, wheezing, chest tightness, cough, sputum production increase, change of color and/or viscosity of sputum, and fever are the main presentations of AECOPD. Some nonspecific symptoms such as tachycardia, insomnia, lethargy, fatigue, depression, and mental disorders can also emerge. When a patient suffers from fever, radiographic abnormalities of the chest, or a decrease in exercise tolerance, AECOPD should be considered. An increase in sputum production and purulent sputum usually suggest bacterial infection. ${ }^{13}$

\section{Diagnosis}

The diagnosis of AECOPD relies exclusively on the clinical presentations of the patients complaining of an acute change of symptoms (baseline dyspnea, cough, and/or sputum production) that is beyond normal day-to-day variation. No single biomarker has been applied in the clinical diagnosis and assessment of AECOPD so far. In the future, a biomarker or group of biomarkers that enable a more precise etiologic diagnosis would be desirable. ${ }^{14}$

\section{Differential diagnosis}

The therapeutic effects for $10 \%-30 \%$ patients with severe AECOPD are not satisfactory, and these patients should be reassessed. Some other confusing diseases like pneumonia, congestive heart failure, pneumothorax, pleural effusion, PE, or arrhythmia should be considered. The poor compliance to treatment also causes a change of symptoms, which can be difficult to distinguish from a real exacerbation. Acute dyspnea of congestive heart failure can be differentiated by the increase of plasma B-type natriuretic peptide associated with other clinical manifestations. ${ }^{1,15}$

\section{Severity of AECOPD}

The assessment of AECOPD is based on the disease course, symptoms, signs, spirometry, blood gas, and other laboratory tests (Table 1). Specific attention should be paid to duration of worsening or new symptoms, severity and frequency of dyspnea and cough, volume and color of sputum, limitation of daily activities, edema, number of previous exacerbations and hospitalizations, and current treatment regimen. It is important to compare with previous laboratory tests so that acute changes can be easily noted. Mental status is an indicator of deterioration, and critical illness needs emergency treatment. Use of accessory respiratory muscles, paradoxical chest wall movements, cyanosis, lower limb edema, right heart failure, and hemodynamic instability also help to tell the severity of AECOPD. For very severe COPD, once mental status changes, the patient should be sent to hospital immediately. ${ }^{1,13}$

\section{Laboratory findings Regular laboratory test}

Red blood cell count and hematocrit may identify polycythemia and bleeding. White blood cell count may help to identify pulmonary infection. White blood cell count increases with or without neutrophil shift to the left in some patients with aggravating infection.

Table I Assessment of AECOPD: medical history and signs

\begin{tabular}{ll}
\hline Medical history & Signs \\
\hline Grading of FEV & $\begin{array}{l}\text { Use of accessory } \\
\text { respiratory muscles } \\
\text { Paradoxical chest wall } \\
\text { Duration of worsening or }\end{array}$ \\
new symptoms & Worsening or new onset \\
Number of previous exacerbations & central cyanosis \\
(acute exacerbations/hospitalizations) & Development of peripheral \\
Comorbidities & edema \\
Current treatment regimen in stable phase & Hemodynamic instability \\
Previous use of mechanical ventilation & Signs of right heart failure \\
& Deteriorated mental status \\
\hline
\end{tabular}

Note: Data from Vestbo J, Hurd SS, Agustí AG, et al. Global strategy for the diagnosis, management, and prevention of chronic obstructive pulmonary disease: GOLD executive summary. Am J Respir Crit Care Med. 2013;187(4):347-365.

Abbreviations: AECOPD, acute exacerbation chronic obstructive pulmonary disease; $\mathrm{FEV}_{\mathrm{I}}$, forced expiratory volume in I second. 


\section{Chest X-ray}

Complications like pleural effusion, pneumothorax, and pneumonia should first be identified by X-ray when consulting an AECOPD patient. Chest radiographs are also useful in distinguishing other diseases presenting similar symptoms, such as pulmonary edema and pleural effusion.

\section{Blood gas test}

The measurement of arterial blood gases is vital for inpatients, to determine the severity of AECOPD. Respiratory failure is indicated when partial pressure of oxygen in arterial blood $\left(\mathrm{PaO}_{2}\right)<60 \mathrm{mmHg}$ or partial pressure of $\mathrm{CO}_{2}$ in arterial blood $\left(\mathrm{PaCO}_{2}\right)>50 \mathrm{mmHg}$ at sea level. Intensive care unit (ICU) admission and monitoring are needed when $\mathrm{PaO}_{2}<50 \mathrm{mmHg}, \mathrm{PaCO}_{2}>70 \mathrm{mmHg}$, and $\mathrm{pH}<7.300^{1,13}$

\section{Spirometry}

$\mathrm{FEV}_{1}<1 \mathrm{~L}$ predicts severe impairment of pulmonary function. Spirometry is not recommended during an exacerbation because it can be difficult to perform and measurements are usually not accurate. ${ }^{1}$

\section{Electrocardiography and echocardiography}

Electrocardiography and echocardiography may aid in the diagnosis of right ventricular hypertrophy, arrhythmia, and myocardial ischemia.

\section{Biochemical test}

Abnormalities including electrolyte disturbances (hyponatremia, hypoglycemia, and hypochloremia), diabetic crisis, or malnutrition (hypoalbuminemia) can be associated with exacerbations. Other metabolic acid-base disturbance can also be found.

\section{Sputum culture and antibiotic sensitivity test}

For patients with purulent sputum during an exacerbation, qualified sputum should be collected for smear, culture, and sensitivity test before starting empirical antibiotic treatment. If an infectious exacerbation does not respond to the initial antibiotic treatment, the results of sputum culture and antibiotic sensitivity tests can guide the medication. Bacteria colonizing in the pharynx can confuse interpretation of microbiological results. In Global Initiative on Obstructive Lung Disease (GOLD) 3 and 4 patients, P. aeruginosa becomes important. Fungal infection should be noticed if the patients have long-term use of antibiotics and systemic corticosteroids, especially for those who have recently suffered from repeated attacks of AECOPD.
For severe AECOPD patients who are likely to be infected by refractory pathogens (eg, P. aeruginosa) or drug-resistant pathogens (seen in those with long-term use of antibiotics or oral corticosteroids, protracted course, more than four exacerbations per year, or $\mathrm{FEV}_{1}<30 \%$ ), trachea secretions from patients with mechanical ventilation or samples collected by bronchoscopy with a protected specimen brush from peripheral airways are recommended for microbial detection. ${ }^{1,9,13}$

\section{Treatment setting of AECOPD}

The goals of treatment for AECOPD are to minimize the impact of the current exacerbation and to prevent the development of subsequent exacerbations. Depending on the severity of an AECOPD and/or the severity of the underlying disease, an exacerbation can be managed in an outpatient or inpatient setting. When a patient comes to the emergency department, the first actions are to provide supplemental oxygen therapy and to determine whether the exacerbation is life-threatening. If so, the patient should be admitted to the ICU immediately. Otherwise, the patient may be managed in the emergency department or hospitalized.

\section{Indications for hospital admission}

Indications for hospital admission are: 1) a significant increase in intensity of symptoms, such as sudden onset of resting dyspnea; 2) severe underlying COPD; 3) onset of new physical signs (eg, cyanosis and peripheral edema); 4) presence of serious comorbidities (eg, heart failure or newly occurring arrhythmias); 5) failure of an exacerbation to respond to initial medical management; 6) older age; 7) unclear diagnosis; and 8) insufficient medical support.

\section{Indications for ICU admission}

Indications for ICU admission are: 1) severe dyspnea that responds inadequately to initial emergency therapy; 2) changes in mental status (confusion, lethargy, or coma); 3) hypoxemia that is persistent or worsens $\left(\mathrm{PaO}_{2}<5.3 \mathrm{kPa}, 40 \mathrm{mmHg}\right)$ and/ or hypercapnia that is severe/worsening respiratory acidosis ( $\mathrm{pH}<7.25$ ) despite supplemental oxygen and noninvasive ventilation; 4) invasive mechanical ventilation is needed; and 5) hemodynamic instability - need for vasopressors. ${ }^{1,13}$

\section{Treatment setting of AECOPD}

No clinical agreement on severity of AECOPD has been reached. In 2004, American Thoracic Society and European Respiratory Society (ATS/ERS) announced a recommendation of diagnosis and treatment of AECOPD. Severity is classified for purposes of simplicity as follows: Level I, 
ambulatory treatment (treated at home) (Table 2); Level II, severe but not life-threatening AECOPD which requires hospitalization (Table 3); Level III, requires ICU admission (leads to acute respiratory failure) (Table 4). ${ }^{6}$

\section{Treatments of AECOPD Controlled oxygen therapy}

Oxygen therapy is a key component of hospital treatment of AECOPD. Satisfactory oxygenation $\left(\mathrm{PaO}_{2}>60 \mathrm{mmHg}\right.$ or $\mathrm{SaO}_{2}$ [saturated oxygen] $>90 \%$ ) can be easily achieved in patients without severe complications. However, the concentration of inhaled oxygen should not be too high in order to avoid carbon dioxide retention or respiratory acidosis. Oxygen can be delivered by venturi masks or nasal prongs. Venturi masks offer more accurate and controlled delivery of oxygen than nasal prongs but are less likely to be tolerated by the patient. Once oxygen is started, arterial blood gases should be checked 30-60 minutes later to ensure satisfactory oxygenation without carbon dioxide retention or respiratory acidosis. ${ }^{1,13}$

\section{Bronchodilators}

Inhaled short-acting $\beta_{2}$-agonists or short-acting $\beta_{2}$-agonists combined with short-acting anticholinergics are usually preferred bronchodilators in AECOPD (Evidence C). (Process of evidence grading [Table S1] as per the GOLD 2013 revision was used.) Clinical symptoms and pulmonary function can be improved by these drugs. Nebulized inhalation with shortacting bronchodilators is especially suitable for AECOPD patients. The effect of a long-acting bronchodilator with or without inhaled corticosteroids is not certain in AECOPD. The adverse effect of theophylline is commonly seen and is only to be used in selected cases when there is insufficient response to short-acting bronchodilators (Evidence B). ${ }^{1}$

Table 2 Management of Level I patients

Patient education
Check inhalation technique
Consider use of spacer devices
Bronchodilators
Short-acting $\beta 2$-agonists and/or ipratropium MDI with spacer or hand-
held nebulizer as needed. Consider adding long-acting bronchodilator
Corticosteroids (the actual dose may vary)
Prednisone $30-40$ mg orally per day for $10-14$ days; consider using an
inhaled corticosteroid
Antibiotics
May be initiated in patients with altered sputum characteristics
Choice should be based on local pattern of bacterial resistance

Note: Data from Chinese Expert Panel on Antibiotics Therapy in Acute Exacerbation of Chronic Obstructive Pulmonary Diseases. The role of antibiotics therapy in guideline for diagnosis and management of chronic obstructive pulmonary diseases. Chin J Tuberc Respir Dis. 2013;36(9):712-7|4."

Abbreviation: MDI, metered-dose inhaler.
Table 3 Management of hospitalized patients

Administer supplemental oxygen therapy and obtain serial arterial blood gas measurement

Bronchodilators

Increase doses and/or frequency of short-acting bronchodilators Combine short-acting $\beta 2$-agonists with anticholinergics

Use spacers or air-driven nebulizers

Add oral or intravenous corticosteroids

Consider antibiotics (oral or occasionally intravenous) when there are signs of a bacterial infection

Consider noninvasive mechanical ventilation

At all times

Monitor fluid balance and nutrition

Consider subcutaneous heparin or low-molecular-weight heparin

Identify and treat associated conditions (eg, heart failure and

arrhythmias)

Closely monitor condition of the patient

Note: Data from Vestbo J, Hurd SS, Agustí AG, et al. Global strategy for the diagnosis, management, and prevention of chronic obstructive pulmonary disease: GOLD executive summary. Am J Respir Crit Care Med. 2013;187(4):347-365.'

\section{Inhaled short-acting bronchodilators}

Inhaled short-acting $\beta_{2}$-agonists with or without short-acting anticholinergics are commonly used for the treatment of AECOPD. $\beta_{2}$-agonists are preferred bronchodilators; shortacting $\beta_{2}$-agonists are more suitable for AECOPD. If the effect is not satisfactory, anticholinergics (eg, ipratropium bromide) are recommended to be added. Inhalation is the preferred

Table 4 Management of patients admitted to ICU

Oxygen therapy, supplemental oxygen, or ventilatory support

Bronchodilators

Short-acting $\beta 2$-agonists, ipratropium, or ipratropium compound with nebulizer

If the patient is on the ventilator, consider MDI administration Corticosteroids

If the patient tolerates oral medications, prednisone $30-40 \mathrm{mg}$ orally per day for 10-14 days

If patient cannot tolerate oral medications, give the equivalent dose intravenously for up to $10-14$ days

Consider using inhaled corticosteroids by MDI or hand-held nebulizer

Antibiotics (based on local bacterial resistance patterns)

Amoxicillin/clavulanate, respiratory fluoroquinolinones (levofloxacin, moxifloxacin)

If Pseudomonas aeruginosa and/or other Enterobacteriaceae are suspected, consider combination therapy

Ciprofloxacin and/or other beta-lactam antibiotics against

Pseudomonas aeruginosa, and aminoglycosides can also be used as a

component of combination therapy

At all times

Monitor fluid balance and nutrition

Consider subcutaneous heparin or low-molecular-weight heparin Identify and treat associated conditions (eg, heart failure and arrhythmias) Closely monitor condition of the patient

Note: Data from Vestbo J, Hurd SS, Agustí AG, et al. Global strategy for the diagnosis, management, and prevention of chronic obstructive pulmonary disease: GOLD executive summary. Am J Respir Crit Care Med. 2013;187(4):347-365.'

Abbreviations: ICU, intensive care unit; MDI, metered-dose inhaler. 
approach for short-acting $\beta_{2}$-agonists and anticholinergics. For AECOPD patients, severe dyspnea, ataxia, and dysesthesia make inhalation difficult; therefore, pressure sprayers are more appropriate. If the pressure sprayer is air driven, hypoxemia could be worsening; however, high inhaled oxygen concentration should be avoided when the sprayer is oxygen driven. If patients are on mechanical ventilation, an adaptor can be used for inhalation. The dosage needs to be 2-4 times higher because of the deposition of drugs in the ventilator circuit. ${ }^{16,17}$ Commonly used short-acting bronchodilator solutions for nebulization include: 1) salbutamol sulfate solution for inhalation, which can be administered by ventilation or nebulizer and diluted with normal saline by sprayers for adults; 2) ipratropium bromide solution for inhalation, which can be administered by nebulizer $(6-8 \mathrm{~L} / \mathrm{min}$ flow rate of oxygen is recommended when oxygen utilities are available; the dosage should be personalized); and 3) ipratropium bromide compound solution, which can be given through a nebulizer or intermittent positive pressure ventilator for adults (the aged included) and teenagers over 12 years old. ${ }^{16}$

\section{Intravenous methylxanthines} (theophylline or aminophylline)

Intravenous methylxanthines are considered second-line therapy, only to be used in selected cases when there is insufficient response to short-acting bronchodilators or in some severe AECOPD patients. The dilation effect of methylxanthines is weaker than $\beta_{2}$-agonists and anticholinergics, but they can be added when illness has not been relieved after 12-24 hours of $\beta_{2}$-agonist or anticholinergic treatment. In addition to a bronchial dilation effect, methylxanthines can also improve the function of respiratory muscles, increase cardiac output, decrease the resistance of the pulmonary circulation, excite the central nervous system, and have an anti-inflammatory effect. In addition, resistance of corticosteroids can be relieved by theophylline. Nevertheless, due to the individual differences of plasma-drug concentration and narrow therapeutic index, it is essential to monitor plasma levels for evaluation of therapeutic effects and avoidance of adverse effects. Twenty-four hours after theophylline initiation, blood concentration should be tested, and dose adjustment should be based on its effective blood concentration range. Given the cardiovascular and nervous toxicity and mortality increase with overdose, theophylline is recommended for low-dose use. ${ }^{13}$

Due to different mechanisms, pharmacokinetic characteristics, and targeted regions of airway, a combination of $\beta_{2}$-agonists, anticholinergics, and theophylline can be more effective than single medication on bronchodilation.

\section{Corticosteroids}

Systemic corticosteroids in hospitalized AECOPD patients can shorten recovery time, improve lung function $\left(\mathrm{FEV}_{1}\right)$ and arterial hypoxemia (Evidence A), and reduce the risk of early relapse and treatment failure, and reduce the length of hospital stay. A dose of 30-40 mg prednisolone per day for 10-14 days is recommended (Evidence D). Therapy with oral prednisolone is preferable rather than intravenous treatment. ${ }^{1,13}$ Systemic prednisone $40 \mathrm{mg}$ per day for 5 days has been shown to be non-inferior to 14-day treatment with regard to re-exacerbation within 6 months while reducing corticosteroid exposure. ${ }^{18}$

Nebulized budesonide suspension for inhalation alone may be an alternative to oral corticosteroids. Time of nebulization and dosage output depend on flow rate, volume of nebulizer, and solution. Nebulized budesonide alone cannot release airflow limitation immediately, so it should be combined with short-acting bronchodilator. ${ }^{1,16}$ Eight milligrams of nebulized budesonide is equal to $40 \mathrm{mg}$ of systemic prednisolone.

\section{Antibiotics}

\section{Indication for antibiotics}

Although the infectious agents in AECOPD can be viral or bacterial, the use of antibiotics in exacerbations remains controversial. There is evidence supporting the use of antibiotics in AECOPD when patients have: 1) three cardinal symptoms - increase in dyspnea, sputum volume, and sputum purulence (Evidence B); 2) two of the cardinal symptoms, if increased purulence of sputum is one of symptoms (Evidence C); or 3) a severe AECOPD attack which requires mechanical ventilation (noninvasive or invasive) (Evidence B). ${ }^{7}$ Antibiotics are not recommended in those with two symptoms but no sputum purulence or with one symptom alone., ${ }^{1,19}$

\section{Categories of antibiotics}

The choice of antibiotics should be based on the local bacterial resistance pattern. In patients with frequent exacerbations, severe airflow limitation, and/or exacerbations requiring mechanical ventilation, cultures from sputum or other materials from the lung should be performed, as Gramnegative bacteria (eg, Pseudomonas spp. or other resistant pathogens) that are not sensitive to the abovementioned antibiotics may be present. For hospitalized patients, culture of sputum or secretion collected by tracheal suction 
(in patients on mechanical ventilation) rather than bronchoscopy is the better way for assessment of bacteria load and potential pathogens.

\section{Route and duration of antibiotics administration}

The route of administration (oral or intravenous) depends on the ability of the patient to eat and the pharmacokinetics of the antibiotic, although preferably antibiotics are given orally. Improvements in dyspnea and sputum purulence suggest clinical effectiveness. The recommended duration of antibiotic treatment is 5-10 days, which can be extended in some special cases (Evidence D).

\section{Recommendations for initiated antibiotic therapy}

AECOPD patients can be divided into two groups: patients with no risk of $P$. aeruginosa infection; and patients at high risk of $P$. aeruginosa infection. If one of the following risk factors appears, $P$. aeruginosa infection should be considered: 1 ) recent hospitalization; 2 ) frequent ( $>4$ times per year) or recent (in the previous 3 months) use of antibiotics; 3 ) severe illness $\left(\mathrm{FEV}_{1}<30 \%\right)$; or 4$)$ oral corticosteroids (prednisone $>10 \mathrm{mg} / \mathrm{d}$ in the previous 2 weeks). ${ }^{9,10}$

For patients without risk factors for $P$. aeruginosa infection, there are several choices of antibiotics, which should be selected based on the severity of exacerbation, local bacterial resistance pattern, cost, and patient compliance. Antibiotics such as amoxicillin/clavulanic acid, levofloxacin, and moxifloxacin are recommended for those with no risk factors of $P$. aeruginosa infection.

In patients with risk factors for $P$. aeruginosa, ciprofloxacin or levofloxacin can be chosen when the oral route is available. When parenteral treatment is needed, ciprofloxacin or a beta-lactam with anti-Pseudomonas aeruginosa activity is available. The addition of aminoglycosides is optional.

The use of the oral or intravenous route should be guided by the stability of clinical condition and the severity of exacerbation. Switch (intravenous to oral) should be done by day 3 of admission if the patient is clinically stable. ${ }^{9,10}$

\section{Therapeutic effect of initial antibiotics treatment}

The effect of antibiotics for AECOPD include short-term effects (ie improving symptoms and lung function, shortening recovery time) and long-term effects (ie minimizing the risk of future exacerbations, reducing bacteria load in the airways). Fungal infections should be noticed, and preventive measures should be applied as necessary for those undergoing treatment with long-term broad-spectrum antibiotics and corticosteroids.
Ten to twenty percent of AECOPD patients may show poor response to initial empirical antibiotic treatment. These failures may be due to the following factors: 1) initial antibiotics have not covered causative pathogens such as $P$. aeruginosa, $S$. aureus (including methicillin-resistant S. aureus), Acinetobacter, and other non-fermenting bacteria; 2) long-term use of corticosteroids puts patients at high risk of fungal infections; 3 ) the causative bacteria is the highly resistant S. pneumoniae; or 4) secondary nosocomial infections in patients on invasive mechanical ventilation.

The underlying reasons need to be analyzed for these patients. Factors commonly seen include inappropriate drug use and other noninfectious factors such as PE and heart failure. Strategies include: 1) trying to identify noninfectious factors; 2) reassessing the possible pathogens; 3 ) changing to other antibiotics which cover $P$. aeruginosa, resistant $S$. pneumonia, and non-fermenting bacteria; or 4) adjusting the treatment according to microbiological test results. ${ }^{9,10}$

\section{Empirical antiviral therapy}

Antiviral agents are not recommended in the treatment of AECOPD, although viral infections play a key role in exacerbation etiology, especially rhinovirus. Many antiviral agents have been tried in the treatment of rhinovirus infections. Compounds have attempted to target cell susceptibility, viral attachment and receptor blockade, viral uncoating, viral RNA (ribonucleic acid) replication, and viral protein synthesis. Unfortunately, although the neuraminidase inhibitors amantadine and zanamivir are effective against influenza, other agents have failed to demonstrate a clinically significant benefit in trials and are often complicated by adverse events and lack of tolerability. ${ }^{7,20}$ Currently, no drugs are licensed for treatment of rhinovirus infections, and thus no antiviral agents are recommended for treating rhinovirus-induced COPD exacerbations. ${ }^{21}$

\section{Empirical use of antiviral treatment and time of onset in AECOPD patients suspected of influenza infection}

According to guidelines for the management of adult lower respiratory tract infections released by the ERS in 2011, the empirical use of antiviral treatment in patients suspected of suffering from influenza is usually not recommended. Only in high-risk patients who have typical influenza symptoms (fever, muscle ache, general malaise, and respiratory tract infection) for $<2$ days, and during a known influenza epidemic, can antiviral treatment be considered., ${ }^{9,10}$ 


\section{Respiratory stimulants}

Respiratory stimulants are not recommended for acute respiratory failure in AECOPD. ${ }^{1}$ Doxapram may be used while the patient is transferred to an area where noninvasive mechanical ventilation can be started.

\section{Adjunct therapies}

Depending on the clinical condition of the patient, an appropriate fluid and electrolyte balance with special attention to their supplementation should be considered; enteral or intravenous nutrition should be provided for those who have eating problems; drainage of sputum (eg, stimulating cough, patting the back, and postural drainage) is encouraged; comorbidities (eg, coronary heart disease, diabetes mellitus, and hypertension) and complications (eg, shock, disseminated intravascular coagulation, and upper gastrointestinal hemorrhage) should be recognized and treated. ${ }^{13}$

\section{Mechanical ventilation}

The goals of mechanical ventilation in AECOPD with respiratory failure include: 1) to relieve hypoxemia, increase $\mathrm{PaO}_{2}$ (with a target saturation over 90\%), and increase oxygen supply to vital organs; 2) to improve acute respiratory acidosis and correct acute life-threatening hypercapnia (without rushing to decrease $\mathrm{PaCO}_{2}$ to normal range); 3) to relieve respiratory distress - dyspnea would be alleviated when primary diseases are relieved; 4) to reduce respiratory muscle fatigue; and 5) to decrease myocardial and total oxygen consumption - the application of mechanical ventilation can decrease myocardial and total oxygen consumption when AECOPD patients suffer from dyspnea, overuse of respiratory and other muscles, disorders of oxygen release, or cardiac overload. ${ }^{22}$

\section{Indications and relative contraindications for noninvasive mechanical ventilation (NIV)}

Table 5 summarizes the indications and relative contraindications of NIV for AECOPD patients who suffer from acute or chronic respiratory failure. NIV has been shown to rectify acute respiratory acidosis, increase $\mathrm{pH}$ value, decrease $\mathrm{PaCO}_{2}$, and decrease the respiratory rate and severity of breathlessness. Consequently, NIV can reduce: the length of hospital stay, mortality, and the need for intubation (Evidence A).

\section{Connection between ventilator and patient}

Comfort, air leakage, and stability have a great influence on therapeutic effect and patients' tolerance. Mask fit is therefore important to ensure effective ventilator support. In addition
Table 5 Indications and relative contraindications for NIV

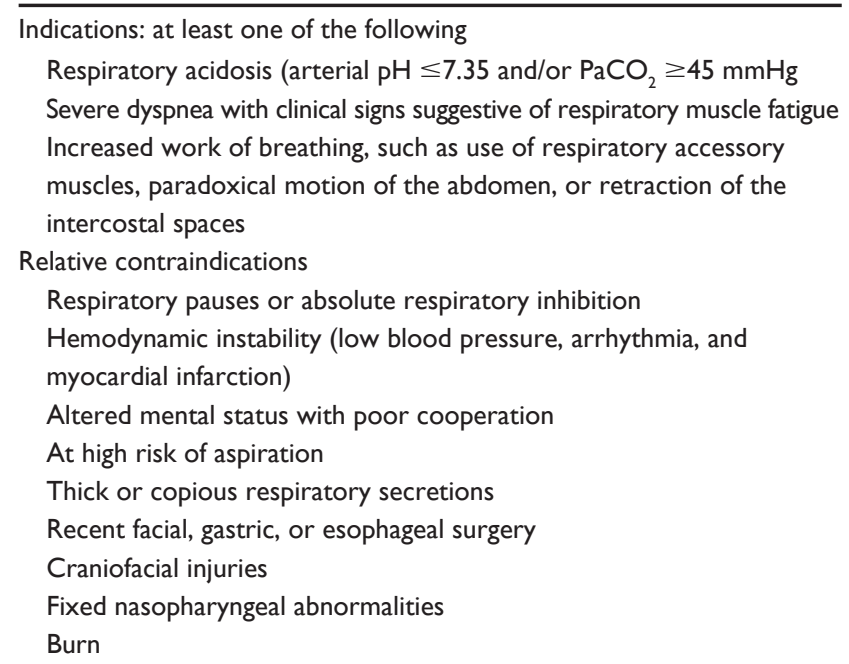

Abbreviations: NIV, noninvasive mechanical ventilation; $\mathrm{PaCO}_{2}$, partial pressure of $\mathrm{CO}_{2}$ in arterial blood.

to a selection of various sizes of nasal masks and oral-nasal masks, tightness of head gear should be individually adjusted to reduce leakage and prevent skin ulceration. ${ }^{22}$

\section{Modes and parameters of ventilation}

Modes commonly used for NIV include continuous positive airway pressure, pressure-controlled ventilation/volumecontrolled ventilation, proportional assisted ventilation (PAV), and pressure support ventilation (PSV) + positive-end expiratory pressure (PEEP), which is usually called bi-level positive airway pressure. Of these, bi-level positive airway pressure is the most often used. Adaptive adjustment of parameters suggests: expiratory positive airway pressure starts from 2 to $4 \mathrm{~cm}$ $\mathrm{H}_{2} \mathrm{O}$ and increases gradually until ventilators show good trigger sensitivity for each inspiration; inspiratory positive airway pressure can start from 4 to $8 \mathrm{~cm} \mathrm{H}_{2} \mathrm{O}$ and increases when the patient has been tolerated until the ventilation is satisfied or the highest ventilation tolerated has been reached. ${ }^{23-25,27}$

\section{Monitoring of NIV in AECOPD:Table 6}

Indications for invasive mechanical ventilation

For AECOPD patients, early intervention of NIV can significantly reduce the use of invasive mechanical ventilation. Patients with severe respiratory failure should be considered for intubation and invasive mechanical ventilation as soon as possible if NIV fails or contraindications exist. Table 7 summarizes indications of invasive mechanical ventilation for AECOPD with respiratory failure. ${ }^{1}$

\section{Ventilation modes}

Modes commonly used include assist/control ventilation, synchronized intermittent mandatory ventilation (SIMV) 
Table 6 Clinical evaluation of NIV in AECOPD

\begin{tabular}{|c|c|}
\hline Vital signs & General state and change of mental status \\
\hline Respiratory system & $\begin{array}{l}\text { Severity of dyspnea, respiratory rate, chest } \\
\text { wall movement, accessory respiratory } \\
\text { muscle recruitment, breath sounds, patient- } \\
\text { ventilation interfaces }\end{array}$ \\
\hline Circulatory system & Heart rate, heart rhythm, and blood pressure \\
\hline Ventilation parameters & $\begin{array}{l}\text { Tidal volume, pressure, frequency, inspiratory } \\
\text { time, and air leakage }\end{array}$ \\
\hline $\begin{array}{l}\text { Blood gas and } \mathrm{SpO}_{2} \\
\text { Sputum drainage }\end{array}$ & $\begin{array}{l}\mathrm{SpO}_{2}, \mathrm{pH}, \mathrm{PaCO}_{2} \text {, and } \mathrm{PaO}_{2} \\
\text { Keep close observation on patient's } \\
\text { expectoration; mask should be removed } \\
\text { regularly for sputum drainage and } \\
\text { expectoration according to patient's illness } \\
\text { and sputum volume }\end{array}$ \\
\hline Adverse effects & $\begin{array}{l}\text { Flatulence, aspiration, mask compression, } \\
\text { dry mouth and nasopharynx, skin damage, } \\
\text { sputum drainage disorder, intolerance, fear } \\
\text { (claustrophobia), barotrauma }\end{array}$ \\
\hline
\end{tabular}

Abbreviations: AECOPD, acute exacerbation chronic obstructive pulmonary disease; NIV, noninvasive mechanical ventilation; $\mathrm{PaCO}_{2}$, partial pressure of $\mathrm{CO}_{2}$ in arterial blood; $\mathrm{PaO}_{2}$, partial pressure of oxygen in arterial blood; $\mathrm{SpO}_{2}$, pulse oximeter oxygen.

and PSV. Other new modes like PAV can also be employed. SIMV + PSV and PSV are generally used. The application of PAV is promising and needs to be further investigated (Table 8).

\section{Parameters}

Dynamic pulmonary hyperinflation (DPH) and intrinsic PEEP (PEEPi) are known as the main mechanical changes of breathing leading to respiratory failure in AECOPD. To relieve these conditions, expiration volume can be increased by restricting tidal volume and respiratory rate and increasing inspiratory flow rate. Besides, to cope with PEEPi, an appropriate level of extrinsic PEEP (PEEPe) can be use to facilitate inspiratory trigger and improve synchrony.

Table 7 Indications for invasive mechanical ventilation

Unable to tolerate NIV or NIV failure

Respiratory or cardiac arrest

Respiratory pauses with loss of consciousness or gasping for air

Diminished consciousness, psychomotor agitation inadequately

controlled by sedation

Massive aspiration

Persistent inability to remove respiratory secretions

Heart rate $<50 \mathrm{~min}^{-1}$ with loss of alertness

Severe hemodynamic instability without response to fluids and

vasoactive drugs

Severe ventricular arrhythmias

Life-threatening hypoxemia in patients unable to tolerate NIV

Note: Data from Vestbo J, Hurd SS, Agustí AG, et al. Global strategy for the diagnosis, management, and prevention of chronic obstructive pulmonary disease: GOLD executive summary. Am J Respir Crit Care Med. 2013;187(4):347-365.'

Abbreviation: NIV, noninvasive mechanical ventilation.
Table 8 Invasive mechanical ventilation in AECOPD with respiratory failure

\begin{tabular}{ll}
\hline Ventilation route & Endotracheal intubation or tracheotomy \\
Ventilation mode & Assist/control ventilation \\
& or Synchronized intermittent mandatory \\
& ventilation \\
& or Pressure support ventilation \\
& To improve gas exchange and relax accessory \\
Goals & respiratory muscles \\
Recommended settings & Tidal volume: $7-9 \mathrm{~mL} / \mathrm{kg}$ \\
& Respiratory rate: $10-15$ breaths/minute \\
& I:E: I:2/I:3 \\
& Inspiratory flow rate $>60 \mathrm{~L} / \mathrm{min}$ \\
& Proper FiO to achieve $\mathrm{SaO} \mathrm{O}_{2}>90 \%$ \\
& Minimized PEEPe \\
& Expiratory end Pplat $<30 \mathrm{~cm} \mathrm{H}_{2} \mathrm{O}$ \\
& Permissive hypercapnia if necessary \\
& Complications due to intubation and \\
& tracheotomy \\
& Risk of alveolar hyperinflation and barotrauma \\
& Lack in activities
\end{tabular}

Abbreviations: $A E C O P D$, acute exacerbation chronic obstructive pulmonary disease; $\mathrm{FiO}_{2}$, fraction of inspired oxygen; I:E, inspiratory to expiratory time ratio; PEEPe, extrinsic positive-end expiratory pressure; Pplat, plateau pressure; $\mathrm{SaO}_{2}$, saturated oxygen.

The setting of ventilator parameters are briefly introduced as follows:

1. Tidal volume or airway pressure: $7-9 \mathrm{~mL} / \mathrm{kg}$ of tidal volume, or plateau pressure less than $30 \mathrm{~cm} \mathrm{H}_{2} \mathrm{O}$ with or without airway peak pressure less than $35-40 \mathrm{~cm} \mathrm{H}_{2} \mathrm{O}$ should be applied to avoid further deterioration of DPH and barotrauma; in addition, appropriate respiratory rate should be set to ensure basic minute ventilation, so that $\mathrm{PaCO}_{2}$ decreases gradually to within the range of stable COPD and alkalosis caused by rapid $\mathrm{PaCO}_{2}$ change is avoided.

2. Respiratory rate: basic minute ventilation volume and DPH deterioration caused by high respiratory frequency should be considered when setting respiratory rate. Generally, $10-15$ breaths/minute is recommended.

3. Inspiratory flow rate (flow): a high inspiratory flow rate ( $>60 \mathrm{~L} / \mathrm{min}$ or even up to $100 \mathrm{~L} / \mathrm{min}$ ) can improve oxygenation and ventilation-perfusion mismatch. Inspiratory to expiratory time ratio of $1: 2$ or $1: 3$ can prolong time for expiration and meet the ventilation need of AECOPD patients by decreasing respiratory work and improving gas exchange. Decreasing wave, square wave, and sine wave are clinical commonly used patterns.

4. PEEPe: an appropriate PEEPe setting can decrease the difference between airway and alveolar pressures, reduce inspiratory load and respiratory work, and improve coordination with the ventilator. In controlled ventilation, 
PEEPe should be less than $80 \%$ of PEEPi, otherwise DPH will deteriorate.

5. Fraction of inspired oxygen $\left(\mathrm{FiO}_{2}\right)$ : oxygenation of AECOPD can be achieved by low $\mathrm{FiO}_{2}$. Comorbidities with or without complications such as pneumonia, atelectasis, pneumothorax, or heart dysfunction are suggested when higher $\mathrm{FiO}_{2}$ is needed. ${ }^{22}$

\section{Monitoring of invasive ventilation in AECOPD treatment}

Parameters displayed by invasive ventilators reflect dynamic changes of aerodynamics in AECOPD patients. These parameters are as follows.

1. Airway pressure: peak pressure (Ppeak; $<35-40 \mathrm{~cm}$ $\mathrm{H}_{2} \mathrm{O}$ ) and plateau pressure (Pplat; $<30 \mathrm{~cm} \mathrm{H}_{2} \mathrm{O}$ ) need close monitoring to avoid barotrauma. Ppeak increase in AECOPD patients on mechanical ventilation predicts increase of airway resistance and/or DPH deterioration. If Pplat increases simultaneously, DPH is suggested to be the main reason of airway pressure increase.

2. PEEPi: increased airway resistance and minute ventilation volume, and decreased elastic resistance and expiratory duration, contribute to PEEPi formation. PEEPi is indicated by the following clinical symptoms, signs, and respiratory and circulatory statuses: 1) expiratory end constant flow detected by ventilators; 2) signs of inspiratory load increase (eg, three concave sign) and consequent failure of coordination with the ventilator; 3) low blood pressure that cannot be explained by circulatory diseases; and 4) Ppeak and Pplat increase in volume controlled ventilation. Expiration hold and esophageal pressure can help to accurately measure PEEPi.

3. Airway resistance: airway resistance estimates therapeutic effect, such as response to bronchodilator, more accurately compared with airway pressure.

4. Gas exchange monitoring: ventilation parameters can be adjusted based on results of blood gases and exhaled carbon dioxide. $\mathrm{pH}$ value and $\mathrm{PaCO}_{2}$ should be monitored to avoid severe alkalosis due to rapid $\mathrm{PaCO}_{2}$ decrease. ${ }^{19}$ The patient's ability of spontaneous respiration and sputum drainage should be evaluated during invasive ventilation for those with respiratory failure. When pulmonary infection has been controlled, signs and symptoms such as sputum changing from purulent to white mucous, decreased sputum volume, and pulmonary rales indicate that respiratory failure has been improved. If $\mathrm{FiO}_{2}$ is less than $40 \%$, blood gases are nearly normal, and $\mathrm{pH}>7.35$ and $\mathrm{PaCO}_{2}$ $<50 \mathrm{mmHg}$, extubation and NIV can be considered for sequential respiratory support. Sequential treatment of invasive and noninvasive ventilation helps to reduce the incidence of ventilator-associated pneumonia and facilitate early weaning. ${ }^{25,26}$

\section{Management of complications of AECOPD}

Severe AECOPD often coexists with many complications the early diagnosis and treatment of which may have a significant impact on prognosis.

\section{AECOPD complicated with heart failure and arrhythmia}

Right heart failure can be relieved when pulmonary infection is controlled and hypoxia and hypercapnia are improved by bronchodilator and appropriate use of diuretics. Cardiotonics are usually not necessary. For some AECOPD patients with infection controlled, cardiotonics can be considered if heart failure cannot be relieved with diuretics alone or left ventricular dysfunction is complicated.

\section{Diuretics}

Mild or moderate diuretics are usually used to reduce blood volume and pulmonary edema, and thus alveolar ventilation and $\mathrm{PaO}_{2}$ will be improved. Large doses and frequent use of diuretics should be avoided due to the possible consequential thick sputum which is hard to expectorate. Long-term use of diuretics can also cause hypokalemia, which will prompt the renal resorption of hydrogen carbonate and metabolic alkalosis, and cause further respiratory center depression and respiratory deterioration.

\section{Cardiotonics}

Cardiotonics are not often recommended for AECOPD complicated with right heart failure. For those with left ventricular dysfunction, cardiotonics can be used with caution. Long-term hypoxemia leads to poor tolerance to digitalis, which means the therapeutic dose and toxic dose are very close, so poisoning and arrhythmia are more likely to occur. Cardiotonics should be used at a low dose.

\section{Treatment of arrhythmia}

Arrhythmia is usually accompanied in AECOPD patients with acute respiratory failure. Metabolic disorders caused by AECOPD such as infection, hypoxia, hypercapnia, and electrolyte disturbance can provoke arrhythmia, and so can iatrogenic factors (eg, digitalis overdose, sympathomimetics and theophylline use, and right catheterization). Unlike with primary heart diseases, if not life-threatening, the main 
therapy for arrhythmia in AECOPD patients is to identify and correct metabolic causes - hypoxia, hypokalemia, hypomagnesemia, and respiratory acidosis or alkalosis, as well as underlying disease treatment. Once the above causes have been treated, arrhythmia will disappear in most cases. Antiarrhythmic drugs can be prescribed if abnormalities still exist when the causes have been corrected or the triggers cannot be removed. Beta receptor antagonists are usually avoided for their negative impact on pulmonary ventilation function. Selective beta 1 receptor antagonists such as metoprolol or bisoprolol are safe in certain conditions. ${ }^{28-30}$

\section{AECOPD complicated with PE}

COPD is an important risk factor for PE, principally when associated with hospitalization. COPD is an important parameter integrated in prognostic scores such as the Simplified Pulmonary Embolism Severity Index. Patients with AECOPD are more likely to suffer from $\mathrm{PE}$ with a high prevalence of $24.7 \%$. The mortality is nearly $30 \%$ in untreated patients. ${ }^{31}$ When PE is comorbid with AECOPD, the diagnosis for PE could be delayed. If $\mathrm{PE}$ is highly suspected in patients with AECOPD, diagnostic examinations should be performed and anticoagulant therapy should be considered. ${ }^{31,32}$

\section{Etiology}

Hypoxia leads to polycythemia which can increase blood viscosity and platelet dysfunction. AECOPD patients have a high incidence of deep vein thrombosis due to poor cardiac and pulmonary function, limited activity, and being bedridden long term. Nonspecific symptoms and signs like dyspnea and hypoxia, which can be also caused by AECOPD, make it difficult to diagnose PE. Hypotension with or without irreversible low $\mathrm{PaO}_{2}(<60 \mathrm{mmHg})$ by high-flow oxygen delivery usually suggests the possibility of PE.

\section{Diagnosis}

Helical computed tomography and pulmonary angiography are the main methods to diagnose AECOPD complicated with PE. Negative plasma D-dimer is helpful to rule out acute PE with low risk, ie, normal D-dimer is a key factor in excluding PE. Radionuclide perfusion and ventilation imaging has limited value in the diagnosis of PE. If deep vein thrombosis, which is an indicator for anticoagulant therapy has been confirmed, angiography is no longer needed.

\section{Prevention}

For long-term bedridden AECOPD patients with polycythemia or dehydration, whether they have a history of thromboembolic diseases or not, heparin or low-molecularweight heparin should be considered for prevention of PE.

\section{Treatment}

For the treatment of AECOPD complicated with PE, refer to the guideline of diagnosis and treatment of PE in the People's Republic of China ${ }^{33}$ and Chinese expert consensus on the diagnosis and treatment of acute PE. ${ }^{34}$

\section{Palliative care and end-of-life care of AECOPD}

The disease trajectory in COPD is usually marked by a gradual decline in health status and increasing symptoms, punctuated by acute exacerbations that are associated with an increased risk of dying. Progressive respiratory failure, cardiovascular diseases, and malignancies are the primary cause of death in patients with COPD hospitalized for an exacerbation. Mortality varies between $23 \%$ and $80 \%$. For all this reasons, palliative care, end-of-life care, and hospice care are important components of the care of patients with advanced COPD. ${ }^{1}$

Palliative care is generally provided to patients with advanced cancer, and GOLD 2013 for the first time has proposed that palliative care be applied in advanced severe COPD patients. ${ }^{1}$ More than $25 \%$ of medical costs are generated in the last year of life. ${ }^{1}$ Respiratory clinicians should communicate with patients with severe COPD and their families in order to make them fully understand possible critical changes of disease, treatment, and cost. It is recommended to provide the patients and their families with enough time and advices on palliative care before decision-making. Meanwhile, medical expenses will be reduced with therapies that come up to what patients expect and meet their values. Palliative care expands traditional disease-model medical treatment to increase the focus on the goal of preventing and relieving suffering and to support the best possible quality of life for patients. Improving quality of life, optimizing function, helping with decision-making about end-of-life care, and providing emotional and spiritual support to patients are the main goals. Palliative care has been shown to improve quality of life, reduce symptoms, and even prolong survival for patients with advanced diseases..$^{35,36}$

Hospice care can be provided in patients' home or in hospice facilities (such as hospitals or nursing homes) for those with advanced diseases, such as patients with dyspnea at rest and poor responsiveness to bronchodilators, and patients with increasing frequencies of hospitalizations or emergency department visits. ${ }^{1,36}$ 


\section{Hospital discharge and prevention of AECOPD Discharge criteria}

Discharge criteria for AECOPD are: 1) the patient, family, and physician are confident that the patient can manage successfully at home; 2) long-acting bronchodilators, either beta 2 -agonists and/or anticholinergics with or without inhaled corticosteroids can be used, and inhaled short-acting $\beta_{2}$-agonist therapy is required no more frequently than every 4 hours; 3 ) the patient, if previously ambulatory, is able to walk across the room; 4) the patient is able to eat and sleep without frequent awakening due to dyspnea; 5) the patient has been clinically stable for 12-24 hours; 6) arterial blood gases have been stable for 12-24 hours; 7) the patient (or home caregiver) fully understands the correct use of medications; and 8) follow-up and home-care arrangements have been completed (eg, visiting nurse, oxygen delivery, and meal provisions).

An effective long-term home maintenance pharmacotherapy regimen for the stable phase of COPD should be identified when patients are discharged from hospital. Also, the patients should be retrained in inhaler technique, and patient education regarding the role of the maintenance regimen should be further enforced. Furthermore, completion of steroid therapy and antibiotics, if prescribed, should be instructed, and the need for long-term oxygen therapy should be reassessed. A follow-up visit at 4- 8 weeks after discharge should be arranged, and a plan for comorbidity management and follow-up should be provided. ${ }^{1}$

\section{Follow-up}

In the follow-up visit at 6 weeks after discharge, the following items should be assessed: the patient's ability to cope with normal environment; measurement of pulmonary function $\left(\mathrm{FEV}_{1}\right)$; inhaler technique and understanding of recommended treatment regimen; need for long-term oxygen therapy and/or home nebulizer; capacity to do physical activity and activities of daily living; COPD assessment test; modified Medical British Research Council; and status of comorbidities.

Home visits by a community doctor may permit earlier discharge of patients hospitalized with an exacerbation without increasing readmission rates. A management plan can increase the number of appropriate therapeutic interventions and shorten recovery time. For patients who are hypoxemic during an exacerbation, arterial blood gases and/or pulse oximetry should be evaluated prior to hospital discharge and in the following 3 months. If the patient remains hypoxemic, long-term supplemental oxygen therapy may be required.

\section{Prevention}

AECOPD can often be prevented. Smoking cessation, influenza and pneumococcal vaccines, knowledge of current therapy including inhaler techniques, and treatment with long-acting inhaled bronchodilators, with or without inhaled corticosteroids, and possibly, phosphodiesterase-4 inhibitors, are therapies that can reduce the number of exacerbations and hospitalizations. International authorized literatures have documented that inhaled corticosteroid/bronchodilator treatment is strongly recommended for the prevention of AECOPD. ${ }^{37} \mathrm{~N}$-acetylcysteine, a possible antioxidant, is predicted to be therapeutically effective for recurrent exacerbations of COPD. Evidence has shown that carbocisteine and $\mathrm{N}$-acetylcysteine may reduce the numbers of exacerbations in those without inhaled corticosteroid therapy. Use of immunomodulators in COPD can decrease the severity and frequency of exacerbations. Early pulmonary rehabilitation after discharge results in clinically significant improvements in exercise capacity and health status at 3 months as well as in recovery after hospitalization for an exacerbation (Evidence A). On the other hand, symptoms, mental status, effect of bronchodilators, and mortality of COPD patients have all been reported improved by pulmonary rehabilitation. Exercise training (such as walking exercise, upper-limb exercises, or strength training), smoking cessation, nutrition counseling, and education are usually included in rehabilitation programs with a minimum effective length of 6 weeks. The longer the program continues, the more effective the results. In addition, functional status, severity of dyspnea, smoking status, and motivation should be considered when rehabilitation is advised. Patients who are chair-bound (Evidence B), or with Modified Medical Research Council grade 4 dyspnea (Evidence B), or continuing smokers (Evidence B) are less likely to respond to or benefit from these programs. ${ }^{1}$ It should be noted that the methods above may be not fully effective, and new drugs and approaches need to be further investigated for AECOPD prevention ${ }^{38}$ (Table 9).

Although studies have shown that macrolides may prevent AECOPD and improve patient quality of life and clinical symptoms, ${ }^{39}$ GOLD 2013 has pointed out that "continuous use of antibiotics was shown to have no effect on the frequency of exacerbations in COPD. A recent trial of daily azithromycin showed efficacy on exacerbation end-points; however, treatment is not recommended because of an unfavorable balance between benefits and side effects." 
Table 9 Therapies to reduce frequency of exacerbations and hospitalizations

\begin{tabular}{ll}
\hline Drug therapies & Non-drug therapies \\
\hline Inhaled corticosteroids & Smoking cessation \\
Fluticasone, budesonide & Home oxygen therapy \\
Inhaled long-acting bronchodilators & Noninvasive ventilation support \\
Indacaterol, salmeterol, & Pulmonary rehabilitation \\
formoterol, tiotropium & Lung volume reduction surgery \\
Phosphodiesterase-4 inhibitors & \\
Roflumilast & \\
Theophylline & \\
Mucolytics & \\
Ambroxol, erdosteine, carbocistenine & \\
Antioxidant & \\
N-acetylcysteine & \\
Immunomodulators & \\
Vaccines & \\
Influenza and pneumococcal vaccines &
\end{tabular}

\section{Advances in telemedicine and AECOPD}

In the past few decades, telemedicine has become a good way to manage AECOPD by improving patients' access to health care services in remote areas, revolutionizing health care delivery. Interventions including video, telephone, and Internet links among health care professionals using telemonitoring or other systems of remote health care could significantly reduce COPD exacerbations and emergency department visits and improve quality of life in COPD patients. ${ }^{40}$ Other potential positive benefits can be early detection of disease exacerbation and timely intervention, enhancement of adherence to drug treatments, and innovative educational approach with no treatment-related costs to patients. However, data transmission technology, related terminal and platform settings are still far from satisfactory to facilitate telehealthcare delivery. ${ }^{41}$ "Internet of Things" is a recent breakthrough in communication technology, which is expected to enhance the feasibility, efficiency, and acceptance of telemedicine.

\section{Acknowledgments}

This study is supported by funding from the National Key Scientific and Technology Support Program (Collaborative Innovation of Clinical Research for Chronic Obstructive Pulmonary Disease and Lung Cancer, No 2013BAI09B09), The National Key Technology R\&D Program of the 12th National Five-year Development Plan 2012BAI05B01 and Shanghai Committee of Science and Technology (No 12411950100).

\section{Disclosure}

The authors declare no potential conflicts of interest with respect to the research, authorship, and/or publication of this article. The authors received no financial support directly for the research, authorship, and/or publication of this article.

\section{References}

1. Vestbo J, Hurd SS, Agustí AG, et al. Global strategy for the diagnosis, management, and prevention of chronic obstructive pulmonary disease: GOLD executive summary. Am J Respir Crit Care Med. 2013;187(4):347-365.

2. Zhong N, Wang C, Yao W, et al. Prevalence of chronic obstructive pulmonary disease in China: a large, population-based survey. $\mathrm{Am} J$ Respir Crit Care Med. 2007;176(8):753-760.

3. Perera PN, Armstrong EP, Sherrill DL, Skrepnek GH. Acute exacerbations of COPD in the United States: inpatient burden and predictors of costs and mortality. COPD. 2012;9(2):131-141.

4. Chen YH, Yao WZ, Cai BQ, et al. Economic analysis in admitted patients with acute exacerbation of chronic obstructive pulmonary disease. Chin Med J (Engl). 2008;121(7):587-591.

5. Zhu M, Cai B. Costs of the last hospitalization for patients with acute exacerbation of chronic obstructive pulmonary disease and patients with lung cancer. Chin J Tuberc Respir Dis. 2009;32(4):258-261.

6. Celli BR, MacNee W. Standards for the diagnosis and treatment of patients with COPD: a summary of the ATS/ERS position paper. Eur Respir J. 2004;23(6):932-946.

7. Mackay AJ, Hurst JR. COPD exacerbations: causes, prevention, and treatment. Med Clin North Am. 2012;96(4):789-809.

8. Molyneaux PL, Mallia1 P, Cox1 MJ, et al. Outgrowth of the bacterial airway microbiome after rhinovirus eacerbation of chronic obstructive pulmonary disease. Am J Respir Crit Care Med. 2013;188(10): 1224-1231.

9. Woodhead M, Blasi F, Ewig S, et al. Guidelines for the management of adult lower respiratory tract infections - summary. Clin Microbiol Infect. 2011;17 Suppl 6:1-24.

10. Chen M, Liu T, Cai B. Interpretation of guidelines for the management of adult lower respiratory tract infections - summary. Int $J$ Respir. 2012;32(16):1201-1206.

11. Chinese Expert Panel on Antibiotics Therapy in Acute Exacerbation of Chronic Obstructive Pulmonary Diseases. The role of antibiotics therapy in guideline for diagnosis and management of chronic obstructive pulmonary diseases. Chin J Tuberc Respir Dis. 2013;36(9):712-714.

12. Ye F, He L, Cai B, et al. Spectrum and antimicrobial resistance of common pathogenic bacteria isolated from patients with acute exacerbation of chronic obstructive pulmonary disease in mainland of China. Chin Med J (Engl). 2013;126(12):2207-2214.

13. Chinese Chronic Obstructive Pulmonary Diseases Study Group, Chinese Society of Respiratory Diseases. Guideline for diagnosis and management of chronic obstructive pulmonary diseases in China (Revised 2013). Chin J Tuberc Respir Dis. 2013;36(4):255-264.

14. Koutsokera A, Stolz D, Loukides S, Kostikas K. Systemic biomarkers in exacerbations of COPD: the evolving clinical challenge. Chest. 2012;141(2):396-405

15. Bertoletti L, Quenet S, Mismetti P, et al. Clinical presentation and outcome of venous thromboembolism in COPD. Eur Respir J. 2012;39(4): 862-868.

16. Yu S. Inhalation therapy of chronic obstructive pulmonary diseases and bronchial asthma. Chin J Drug App Monitor. 2006;3(4):28-31.

17. Chinese Expert Panel on Inhalation Therapy in Adult Chronic Airway Diseases. Expert consensus on inhalation therapy in adult chronic airway diseases. Chin J Respir Crit Care Med. 2012;11(2):105-110.

18. Leuppi JD, Schuetz P, Bingisser R, et al. Short-term vs conventional glucocorticoid therapy in acute exacerbations of chronic obstructive pulmonary disease: the REDUCE randomized clinical trial. JAMA. 2013;309(21):2223-2231. 
19. Miravitlles M, Anzueto A. Antibiotics for acute and chronic respiratory infection in patients with chronic obstructive pulmonary disease. $\mathrm{Am} \mathrm{J}$ Respir Crit Care Med. 2013;188(9):1052-1057.

20. Mackay AJ, Hurst JR. COPD exacerbations: causes, prevention, and treatment. Med Clin North Am. 2012;96(4):789-809.

21. Mallia P, Singanayagam A, Johnston SL. Virus-bacteria interactions in COPD exacerbations. Eur Respir Monogr. 2013;60:76-83.

22. Chinese Society of Critical Care Medicine. Guideline for mechanical ventilation in patients with acute exacerbation of chronic obstructive pulmonary disease (2007). Chin Crit Care Med. 2007;19(9):513-518.

23. Qin C, Chen R, Zhong N. The physiological impact of and clinical strategy for PEEPi in chronic obstructive pulmonary disease. Chin J Respir Crit Care Med. 2004;3(2):126-128.

24. Luo Q, Chen R. Analysis on treatment compliance of patients with acute exacerbations of chronic obstructive pulmonary disease on noninvasive positive pressure ventilation. Guangdong Medical Journal. 2012;33(9):1310-1312. [Chinese.]

25. British Thoracic Society Standards of Care Committee. Non-invasive ventilation in acute respiratory failure. Thorax. 2002;57(3):192-211.

26. Wang C, Shang M, Huang K. Sequential non-invasive following shortterm invasive mechanical ventilation in COPD induced hypercapnic respiratory failure. Chin J Tuberc Respir Dis. 2000;23(4):212.

27. Chinese Respiratory Physiology and Intensive Care Study Group, Editorial Committee of Chinese Journal of Tuberculosis and Respiratory Diseases. Expert consensus on non-invasive positive pressure ventilation. Chin J Tuberc Respir Dis. 2009;32(2):86-98.

28. Wise AL. Chronic obstructive lung disease: clinical course and management. In: Fishman AP, editor. Fishman's Pulmonary Diseases and Disorders. 4th ed. New York: McGraw-Hill. 2008;729-746.

29. Albert P, Calverley PM. Drugs (including oxygen) in severe COPD. Eur Respir J. 2008;31(5):1114-1124.

30. Ban A, Ismail A, Harun R, et al. Impact of clinical pathway on clinical outcomes in the management of COPD Exacerbation. BMC Pulm Med. 2012;12:27-42.

31. Rizkallah J, Man SF, Sin DD. Prevalence of pulmonary embolism in acute exacerbations of COPD: a systematic review and meta-analysis. Chest. 2009;135(3):786-793.
32. Li X, Wang C, Yang Y. Advances in acute exacerbations of chronic obstructive pulmonary disease complicated with venous thromboembolism. Chinese Journal for Clinicians. 2008;36(7): 26-28. [Chinese.]

33. Chinese Society of Respiratory Diseases. Guidelines for diagnosis and treatment of pulmonary thromboembolism. Chin J Tuberc Respir Dis. 2001;24(5):259-264.

34. Chinese Pulmonary Vascular Diseases Study Group, Cardiovascular Physician Branch. Experts Consensus on Acute Pulmonary Thromboembolism in China. Chin J Intern Med. 2010;49(1):74-81.

35. Brulotte CA, Lang ES. Acute exacerbations of chronic obstructive pulmonary disease in the emergency department. Emerg Med Clin North Am. 2012;30(2):223-247.

36. Liu T, Cai B. Introduction of global strategy for the diagnosis, management and prevention of chronic obstructive pulmonary disease (Revised 2013). Chin J Tuberc Respir Dis. 2013;36(11):805-807.

37. Han MK, Martinez FJ. Pharmacotherapeutic approaches to preventing acute exacerbations of chronic obstructive pulmonary disease. Proc Am Thorac Soc. 2011;8(4):356-362.

38. Zheng JP, Kang J, Huang SG, et al. Effect of carbocisteine on acute exacerbation of chronic obstructive pulmonary disease (PEACE Study): a randomised placebo-controlled study. Lancet. 2008;371(9629): 2013-2018.

39. Llor C, Moragas A, Hernandez S, Bayona C, Miravitlles M. Efficacy of antibiotic therapy for acute exacerbations of mild to moderate chronic obstructive pulmonary disease. Am J Respir Crit Care Med. 2012;186(8):716-723.

40. McLean S, Nurmatov U, Liu JL, et al. Telehealthcare for chronic obstructive pulmonary disease. Cochrane Database Syst Rev. 2011(7):CD007718.

41. Yohannes AM. Telehealthcare management for patients with chronic obstructive pulmonary disease. Expert Rev Respir Med. 2012;6(3): 239-242. 


\section{Supplementary table}

Table SI Description of levels of evidence

\begin{tabular}{ll}
\hline Evidence category & Source of evidence \\
\hline A & $\begin{array}{l}\text { Randomized controlled trials } \\
\text { Rich body of data } \\
\text { B }\end{array}$ \\
Randomized controlled trials \\
C & Limited body of data \\
& Observandomized trials \\
D & Panel consensus judgment \\
\hline
\end{tabular}

International Journal of COPD

\section{Publish your work in this journal}

The International Journal of COPD is an international, peer-reviewed journal of therapeutics and pharmacology focusing on concise rapid reporting of clinical studies and reviews in COPD. Special focus is given to the pathophysiological processes underlying the disease, intervention programs, patient focused education, and self management protocols.

This journal is indexed on PubMed Central, MedLine and CAS. The manuscript management system is completely online and includes a very quick and fair peer-review system, which is all easy to use. Visit http://www.dovepress.com/testimonials.php to read real quotes from published authors. 